\title{
CORRELACIÓN ANALÍTICA-TÉCNICA \\ ENTRE ENSAYOS DE LABORATORIO \\ DE MÓDULO RESILIENTE Y CALIFORNIA \\ BEARING RATIO DE LA MINA COPETO
}

\author{
ANALYTICAL-TECHNICAL CORRELATION BETWEEN \\ LABORATORY TESTS OF RESILIENT MODULE \\ AND CALIFORNIA BEARING RATIO OF THE MINE COPETO
}

Felipe Parra P., Patricio Espinoza-Montero, ${ }^{2}$ Lenys Fernández, ${ }^{2,4}$

Hicher Zambrano, ${ }^{3}$ Wilson Cando ${ }^{1 *}$

Recibido: 30 de septiembre 2019 / Aceptado: 12 de diciembre 2019

DOI: 10.26807/ia.v8i1.123

Palabras clave: California Bearing Ratio, Módulo Resiliente,
correlación CBR-Módulo Resiliente, bases granulares, sub-bases granulares.

Keywords: California Bearing Ratio, Resilient Modulus, correlation

C.B.R. - Resilient Modulus, granular bases, granular sub-bases.

\section{RESUMEN}

Se reporta una correlación analítica-técnica entre ensayos de laboratorio de Módulo Resiliente y California Bearing Ratio, para un agregado que proviene

1 Pontificia Universidad Católica del Ecuador, Facultad de Ingeniería, Escuela de Ingeniería Civil, Quito,

Ecuador (*WOCANDO@puce.edu.ec)

2 Pontificia Universidad Católica del Ecuador, Facultad de Ciencias Exactas y Naturales, Escuela de Química, Quito, Ecuador (Imfernandez@puce.edu.ec, PESPINOZA646@puce.edu.ec)

3 Universidad de Los Andes, Facultad de Ingeniería, Mérida, Venezuela (zhichert@gmail.com)

4 Universidad Simón Bolívar, Caracas, Venezuela (Ifernandez@puce.edu.ec) 
de la mina de Copeto (Toachi - Santo Domingo de Los Tsáchilas-Ecuador), la cual arroja valores reales útiles en la optimización del material utilizado en proyectos viales, a través de la obtención del Módulo Resiliente. El estudio se sustenta en la cualificación de la mina, mediante ensayos fisicoquímicos, que permite conocer las características propias de los agregados, según las normas ASTM. Con el logro de una correlación entre el CBR y el Módulo Resiliente real de la mina en estudio, se tiene valores reales en el comportamiento de los materiales, que se van a usar en la estructura de la vía, lo que genera como resultado espesores de capas menores, que, al momento de la construcción reduce el tiempo de entrega, la cantidad de material y por consiguiente el costo final de la construcción de la vía.

\section{ABSTRACT}

An analytical-technical correlation between laboratory tests of Resilient Module and California Bearing Ratio for an aggregate that comes from the Copeto Mine (Toachi - Santo Domingo de Los Tsáchilas-Ecuador) is reported, which yields real useful values in the optimization of the material used in road projects, through the obtaining of the Resilient Module. The study is based on the qualification of the mine, through physicochemical tests, which allows to know the characteristics of the aggregates that make it up, according to ASTM standards. We report real values in the behavior of the materials that are going to be used in the structure of the road, which results in thicknesses of smaller layers, as in the base (a reduction of $5 \mathrm{~cm}$ ) that, at the time of construction It reduces the delivery time, the amount of material and therefore the final cost of the construction of the road. 


\section{INTRODUCCIÓN}

Esta investigación está orientada a la obtención de una correlación analítica-técnica entre ensayos de laboratorio de C.B.R. (California Bearing Ratio, por sus siglas en inglés) y Módulo Resiliente, aplicados a agregados que provienen de la mina de Copeto ubicada en la provincia de Santo Domingo de los TsáchilasEcuador. Los materiales procedentes de las canteras de esta mina, son utilizados para la conformación de base y sub-base de vías asfálticas, que ayudan a que la capa de rodadura resista las demandas por cargas vehiculares. El espesor de cada capa depende de factores como la cantidad de vehículos en la capa de rodadura, condiciones climáticas, condición del suelo de la sub-rasante y posición del nivel freático.

En la nueva normativa AASHTO1993, 2012, para el diseño y construcción de carreteras, se precisa la necesidad de obtener Módulos Resilientes de estructuras sometidas a cargas dinámicas, esto en la base y sub-base, y de los materiales que van a conformar la estructura del pavimento.
El C.B.R., es una metodología muy utilizada para la caracterización de suelos, así como para conocer el esfuerzo cortante que tiene un material que será utilizado en el diseño de pavimentos controlando la humedad y densidad (Herrera, 2014, Gárnica, 2001). Mientras que, con el Módulo Resiliente, se pueden caracterizar materiales agregados utilizados en la constitución de la sección base y sub-base de la estructura de la vía, para la elaboración de pavimentos (Camacho, 2013). Se ha reportado, la obtención del Módulo Resiliente a través de una correlación con el C.B.R., mediante ecuaciones analíticas (Higuera, 1989). En el presente trabajo, se realizaron ensayos de laboratorio con materiales provenientes de la mina Copeto, aplicando ambas metodologías, a fin de obtener valores reales y específicos de los verdaderos agregados que se utilizan y conforman la estructura de la vía, y así lograr una correlación analíticatécnica más ajustada y exacta de las características fisicoquímicas de los sustratos, lo que resultará en un diseño óptimo de las capas de las vías. Esta metodología no es utilizada en la actualidad en nuestro país; donde 
se realiza únicamente el ensayo de C.B.R. de laboratorio y mediante fórmulas teórica se encuentra el Módulo Resiliente, sin tomar en cuenta que dichas fórmulas no corresponden necesariamente a los tipos de materiales existentes en las minas del Ecuador. En nuestra investigación se han logrado obtener los valores de los ensayos de Módulo Resiliente y C.B.R. de laboratorio de los materiales de base y sub-base de la mina en estudio, logrando obtener datos reales para conformar la correlación, a través de la cual se optimiza el material para el diseño de las capas de la estructura vial.

\section{MATERIALES Y MÉTODOS}

\section{Granulometría del material}

Se realizó para conocer los diferentes tamaños y distribución de las partículas que se ensayaron, con el fin de definir si el agregado cumple con las especificaciones para las clases de base y sub-base (ASTM C33).

Se debe indicar que las granulometrías de las diferentes bases y sub bases son ajustadas a las especificaciones técnicas del Ministerio de Transporte y Obras Públicas (MTOP).

\section{Gravedad Específica y Absorción}

Se determinó para evaluar la relación de vacíos en el agregado, así como el volumen que ocupa en una mezcla y la absorción para conocer el porcentaje de agua que tiene el material (ASTM C128, ASTM C127).

\section{Contenido orgánico del material}

Se evaluó para determinar si existen componentes perjudiciales en arenas naturales (ASTM C40-99).

\section{Terrones de arcilla y partícula fria-} bles del material

Permitió conocer la cantidad de sustancias nocivas y perjudiciales que tiene el agregado (ASTM C142-97).

\section{Durabilidad del agregado a sulfatos}

Permitió conocer el desgaste de los agregados a la acción de los sulfatos, al estar en contacto con el suelo y el agua (ASTM C88). 


\section{Equivalente de arena del material}

Permitió determinar la cantidad de polvo y arcilla en el agregado y limitar la cantidad de este elemento (ASTM D2419).

Durabilidad del agregado a la abrasión

Permitió estimar el desgaste que sufre el agregado grueso en su resistencia y a la fragmentación a la que está expuesto (ASTM C131).

\section{Contenido de humedad del material (según la norma ASTM D4318-84).}

\section{Límites de Atterberg}

Permitió determinar el contenido de agua entre los límites líquido, plástico y semisólido (ASTM D4318-84).

\section{Módulo Resiliente}

Se ensayaron muestras en estado natural y compactadas, a las cuales se les varió el estado de humedad con el fin de determinar la variabilidad del Módulo Resiliente y la deformación bajo cargas cíclicas, impartidas por un pistón servo controlado de un equipo triaxial cíclico. Se aplicó la metodología de ensayo prevista en la norma del Instituto de Vías INV E 156.

\section{RESULTADOS}

\section{Caracterización fisicoquímica de la mina}

La Tabla 1, muestra los parámetros obtenidos en los ensayos de laboratorio para la caracterización de la mina, de acuerdo con el objetivo perseguido en cada análisis y las normas que deben cumplir dichos parámetros.

\section{Valores de C.B.R.}

La Tabla 2, muestra los resultados del ensayo C.B.R. en laboratorio, a partir del cual se evaluó la resistencia potencial al esfuerzo cortante de la base, sub-base, sub-rasante, para el uso en las vías cuando sus condiciones y características van a alterarse en el momento de la construcción. 
InfoANALÍTICA 8(1)

Enero 2020

Tabla 1. Ensayos de Caracterización

\begin{tabular}{|c|c|c|c|c|c|c|c|c|c|}
\hline \multirow{2}{*}{ ENSAYO } & \multirow{2}{*}{ TIPO } & \multirow{2}{*}{ TAME : } & \multirow{2}{*}{ \% PASA } & \multicolumn{2}{|c|}{ CLASIFICACION } & \multirow{2}{*}{ VALOA } & \multirow{2}{*}{ UNIDAD } & \multirow{2}{*}{ ESPECIF. } & \multirow{2}{*}{ OBSERVACIÓN } \\
\hline & & & & sucs & AMSHTO & & & & \\
\hline \multirow{7}{*}{ GRANULOMETRIA } & SUBBASE CLASE 1 & $\begin{array}{c}11 / 2 \\
\text { No. } 4 \\
\text { No. } 40 \\
\text { No. } 200\end{array}$ & $\begin{array}{c}100 \\
39.28 \\
22,26 \\
12.57\end{array}$ & GP-GW & A-1-a & & & $\begin{array}{c}100 \\
30-70 \\
10-35 \\
0-15\end{array}$ & CUMPLE \\
\hline & SUBBASE CLASE 2 & \begin{tabular}{|c|}
2 \\
$11 / 2$ \\
No. 4 \\
No. 40 \\
No. 200 \\
\end{tabular} & $\begin{array}{c}100 \\
94,87 \\
36,98 \\
16,56 \\
5,64 \\
\end{array}$ & GP - GW & A-1-a & & & $\begin{array}{c}100 \\
70-100 \\
30-70 \\
15-40 \\
0-20 \\
\end{array}$ & CUMPLE \\
\hline & SUBBASE CLASE 3 & \begin{tabular}{|c|}
3 \\
$\mathrm{No} .4$ \\
$\mathrm{No} .200$ \\
\end{tabular} & $\begin{array}{c}100 \\
47,16 \\
11,64 \\
\end{array}$ & GP - GW & A-1-a & & & $\begin{array}{c}100 \\
30-70 \\
0-20 \\
\end{array}$ & CUMPLE \\
\hline & BASE CLASE I TIPOA & \begin{tabular}{|c|}
2 \\
$11 / 2$ \\
1 \\
$3 / 4$ \\
$3 \times 1$ \\
No. 4 \\
No. 10 \\
No. 40 \\
No. 200 \\
\end{tabular} & $\begin{array}{c}100 \\
91,83 \\
78,31 \\
72,55 \\
56,31 \\
38,04 \\
27,73 \\
17,10 \\
12,4 \\
\end{array}$ & GP-GW & A-1-a & & & $\begin{array}{c}100 \\
70-100 \\
55-85 \\
50-80 \\
35-70 \\
25-50 \\
20-40 \\
10-25 \\
2-12 \\
\end{array}$ & CUMPLE \\
\hline & BASE CLASE 2 & \begin{tabular}{|c|}
1 \\
$3 / 4$ \\
$3 / 8$ \\
No. 4 \\
No. 10 \\
No. 40 \\
No. 200 \\
\end{tabular} & $\begin{array}{c}100 \\
86,46 \\
64,99 \\
45,69 \\
32,52 \\
17,84 \\
6,43 \\
\end{array}$ & GP-GW & A-1-a & & & $\begin{array}{c}100 \\
70-100 \\
50-80 \\
35-65 \\
25-30 \\
15-30 \\
3-15 \\
\end{array}$ & CUMPLE \\
\hline & BASE CLASE 3 & \begin{tabular}{|c|}
$3 / 4$ \\
No. 4 \\
No. 10 \\
No. 40 \\
No. 200 \\
\end{tabular} & $\begin{array}{c}100 \\
52,02 \\
40 \\
28,16 \\
17,24 \\
\end{array}$ & GP - GW & A-1-a & & & $\begin{array}{c}100 \\
45-80 \\
30-60 \\
20-35 \\
3-15 \\
\end{array}$ & CUMPLE \\
\hline & BASE GLASE 4 & \begin{tabular}{|c|}
2 \\
1 \\
No. 4 \\
No. 200 \\
\end{tabular} & $\begin{array}{c}100 \\
82,99 \\
35,46 \\
8,22 \\
\end{array}$ & GP - GW & A-1-a & & & $\begin{array}{c}100 \\
60-90 \\
20-50 \\
0-15 \\
\end{array}$ & CUMPLE \\
\hline $\begin{array}{c}\text { GRAVEDAD ESPECIFICAY } \\
\text { ABSOACIÓN }\end{array}$ & $\begin{array}{c}\text { AGREGADO FINO (GE) } \\
\text { AGREGADO FINO (ABS) } \\
\text { AGREGADO GRUESO (GE) } \\
\text { AGREGADO GRUESO (ABS) }\end{array}$ & & & & & $\begin{array}{l}2,80 \\
2,50 \\
2,63 \\
1,11 \\
\end{array}$ & $\begin{array}{c}\text { Sin Unidad } \\
\% \\
\text { Sin Unedad } \\
\%\end{array}$ & & \\
\hline CONTENIDO ORGÁNICO & AGREGADO FINO & & & & & COLOA 2 & Sin Unidad & MÁx COLOA 3 & CUMPLE \\
\hline $\begin{array}{l}\text { TERHONES DE APCILLAY } \\
\text { PARTICULAS FRIABLES }\end{array}$ & AGAEGADO FINO & & & & & 0.22 & $\%$ & $\operatorname{Max} .196$ & CUMPLE \\
\hline $\begin{array}{l}\text { DURABULIDAD DE LOS } \\
\text { AGREGADOS A SULFATOS }\end{array}$ & $\begin{array}{l}\text { AGREGADO FINO } \\
\text { AC.AECAADO GAUESO }\end{array}$ & & & & & $\begin{array}{l}5,7 \\
8,7 \\
\end{array}$ & $\begin{array}{l}\text { \% desgaste } \\
\text { \% desgaste } \\
\end{array}$ & $\operatorname{MAX} \cdot 18 \%$ & CUMPLE \\
\hline EOUNALENTE DE NAENA & AGAEGADO FINO & & & & & 81,82 & $\%$ & $>50 \%$ & CUMPLE \\
\hline DUPABI IDAD DEL. & SUBBASE & & & & & 15,12 & \% desgaste & $<50 \%$ & CUMPIE \\
\hline ACAECGADO AL A ABFASIÓN & BASE & & & & & 23.5 & \% desgaste & $<40 \%$ & GUMPLE \\
\hline CONTENIDO DE HUMEDAD & $\begin{array}{l}\text { SUBBASE CLASE } 1 \\
\text { SUBBASE CLASE } 2 \\
\text { SUBBASE CLASE } 3 \\
\text { BASE CLASE 1 THPO A } \\
\text { BASE CLASE } 2 \\
\text { BASE CLASE 3 } \\
\text { BASE CLASE 4 }\end{array}$ & & & & & $\begin{array}{l}24,65 \\
21.34 \\
12,92 \\
20,81 \\
13.57 \\
15,66 \\
19.85 \\
\end{array}$ & $\begin{array}{l}\% \\
\% \\
\% \\
\% \\
\%\end{array}$ & & \\
\hline LiMTIES DE ATTERBERG & AGREGADO FINO & & & & & NP & & & Alto cortenido de arena \\
\hline
\end{tabular}


Tabla 2. Valores de C.B.R.

\begin{tabular}{lcccc}
\multicolumn{1}{c}{ Tipo } & $\begin{array}{c}\text { Densidad } \\
\text { Máxima } \\
\left(\mathrm{g} / \mathrm{cm}^{3}\right)\end{array}$ & $\begin{array}{c}\text { Densidad } \\
\text { Máxima }(\mathbf{9 5 \% )} \\
\left(\mathrm{g} / \mathrm{cm}^{3}\right)\end{array}$ & $\begin{array}{c}\text { Humedad } \\
\text { Optima } \\
(\%)\end{array}$ & $\begin{array}{c}\text { C.B.R. } \\
(\%)\end{array}$ \\
\hline SUBBASE CLASE 1 & 1972 & 1873,4 & 9,81 & 77,8 \\
SUBBASE CLASE 2 & 1988 & 1888,6 & 9,64 & 74,5 \\
SUBBASE CLASE 3 & 1926 & 1829,7 & 10,9 & 70,2 \\
BASE CLASE 1 TIPO A & 2187 & 2077,65 & 10,06 & 99,2 \\
BASE CLASE 2 & 2131 & 2024,45 & 9,95 & 95,2 \\
BASE CLASE 3 & 2079 & 1975,05 & 10,29 & 89,5 \\
BASE CLASE 4 & 2045 & 1942,75 & 9,64 & 86,1 \\
\hline
\end{tabular}

Valores de Módulo Resiliente

Con el fin de obtener mejores resultados, se realizaron tres ensayos de
Módulo Resiliente para cada clase de base y sub-base, los cuales se resumen en la Tabla 3.

Tabla 3. Valores de Módulo Resiliente

\begin{tabular}{|lcccccc|}
\hline \multicolumn{1}{|c}{ Tipo } & $\begin{array}{c}\text { Mr1 } \\
\text { (Mpa) }\end{array}$ & $\begin{array}{c}\text { Mr1 } \\
\text { (psi) }\end{array}$ & $\begin{array}{c}\text { Mr 2 } \\
\text { (Mpa) }\end{array}$ & $\begin{array}{c}\text { Mr 2 } \\
\text { (psi) }\end{array}$ & $\begin{array}{c}\text { Mr 3 } \\
\text { (Mpa) }\end{array}$ & $\begin{array}{c}\text { Mr 3 } \\
\text { (psi) }\end{array}$ \\
\hline SUBBASE CLASE 1 & 279 & 40393 & 286 & 41411 & 290 & 42074 \\
SUBBASE CLASE 2 & 271 & 39334 & 284 & 41127 & 271 & 39321 \\
SUBBASE CLASE 3 & 249 & 36151 & 222 & 32248 & 266 & 38551 \\
BASE CLASE 1 TIPO A & 312 & 45207 & 316 & 45771 & 316 & 45893 \\
BASE CLASE 2 & 305 & 44208 & 308 & 44702 & 306 & 44409 \\
BASE CLASE 3 & 304 & 44083 & 303 & 43912 & 300 & 43567 \\
BASE CLASE 4 & 297 & 43097 & 294 & 42572 & 299 & 43398 \\
\hline
\end{tabular}

Con los resultados obtenidos, utilizando las metodologías el C.B.R. y el Módulo Resiliente, se obtuvo una correlación que permite obtener los valores del Módulo Resiliente (MPa) a través de valores del C.B.R. (\%) con la formulación de una ecuación ana- lítica. Mediante la ecuación, se obtuvieron valores de Módulos Resilientes a partir del valor C.B.R., para bases (Figura 1) y sub-bases (Figura 2) de la mina de Copeto - Santo Domingo de los Tsáchilas 


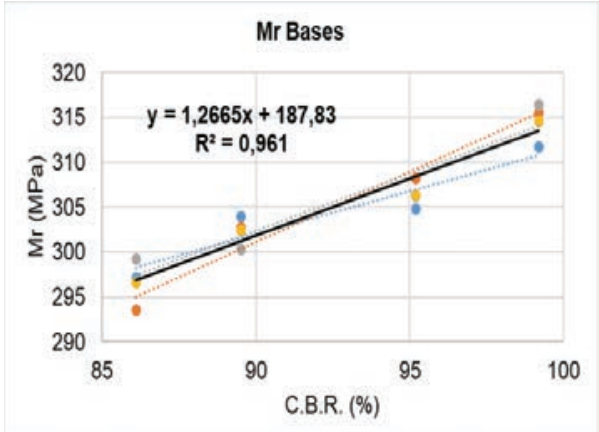

Figura 1. Correlación de Módulo Resiliente para Sub-base

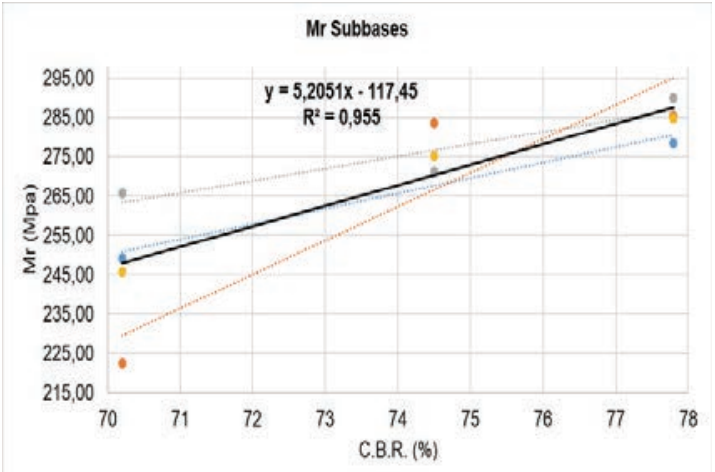

Figura 2. Correlación de Módulo Resiliente para Base

Ecuación para Sub-bases

$M r(M P a)=5.2051 \times C \cdot B \cdot R \cdot(\%)-117.45$
(1)

De acuerdo a la ecuación obtenida para Módulo Resiliente, se ingresa el número C.B.R. (\%) y se obtiene el valor de Mr (Tabla 4). 
Tabla 4. Valores de Módulo Resiliente para Sub-base

\begin{tabular}{|cccc}
\hline Tipo & $\begin{array}{c}\text { C.B.R. } \\
\text { (\%) }\end{array}$ & $\begin{array}{c}\text { Mr } \\
\text { correlación } \\
\text { (Mpa) }\end{array}$ & $\begin{array}{c}\text { Mr } \\
\text { correlación } \\
\text { (psi) }\end{array}$ \\
\hline SUBBASE CLASE 1 & 77.8 & 288 & 41699 \\
SUBBASE CLASE 2 & 74.5 & 270 & 39208 \\
SUBBASE CLASE 3 & 70.2 & 248 & 35962 \\
\hline
\end{tabular}

\section{Ecuación para Bases}

$M r(M P a)=1.2665 \times C . B . R .(\%)+187.83$

Según la ecuación obtenida para Módulo Resiliente, se ingresa el número C.B.R. en \% y se obtiene el valor de Mr (Tabla 5).

Tabla 5. Valores de Módulo Resiliente para Base

\begin{tabular}{lccc}
\hline \multicolumn{1}{c}{ Tipo } & $\begin{array}{c}\text { C.B.R. } \\
\text { (\%) }\end{array}$ & $\begin{array}{c}\text { Mr } \\
\text { correlación } \\
\text { (Mpa) }\end{array}$ & $\begin{array}{c}\text { Mr } \\
\text { correlación } \\
\text { (psi) }\end{array}$ \\
\hline BASE CLASE 1 TIPO A & 99,2 & 313 & 45465 \\
BASE CLASE 2 & 95,2 & 308 & 44730 \\
BASE CLASE 3 & 89,5 & 301 & 43683 \\
BASE CLASE 4 & 86,1 & 297 & 43058 \\
\hline
\end{tabular}

\section{Diseño vial}

Para el diseño de una vía se necesita el TPDA (Tráfico Promedio Diario Anual) inicial y el final (Tabla 6). El período de diseño se realiza con perspectiva de duración de la vía de 10 años.
Tabla 6. Tráfico Promedio Diario Anual inicial y final (TPDA)

\begin{tabular}{cccc}
\hline Tipo & TPDAO & $\%$ & TPDA final \\
\hline 2D & 171 & 52 & 230 \\
2DB & 15 & 5 & 20 \\
3A & 142 & 43 & 191 \\
Total & 328 & & 441 \\
\hline
\end{tabular}

Al ser una vía ubicada en el sector rural del cantón Cayambe, provincia de Pichincha, el nivel de confianza de la vía en diseño fue $\mathrm{C}=90 \%$, la desviación estándar So=0,44, distribución de tráfico Dt $=0,5$, tasa de crecimiento $\mathrm{i}=3 \%$, pérdida por servicio $\Delta \mathrm{psi}=2,0$.

Diseño de las capas de la estructura de la vía por el método AASHTO (93)

La Figura 3, muestra los espesores de las capas de la estructura vial.

\begin{tabular}{|l|c|}
\hline D1 & $=10 \mathrm{~cm}$ \\
D2 & $=20 \mathrm{~cm}$ \\
\cline { 2 - 2 } D3 & $=15 \mathrm{~cm}$
\end{tabular}

Figura 3. Espesores de la estructura vial por el método AASHTO (1993) 
Diseño de las capas de la estructura de la vía por la correlación obtenida

Con la ecuación obtenida, se obtuvo el valor de Módulo Resiliente real del material proveniente de la mina en estudio, es decir que con estos datos reales, de acuerdo a las características del material, se realizó el diseño adecuado de los espesores de la estructura vial (Figura 4).

\begin{tabular}{|l|c|}
\hline D1 & $=10 \mathrm{~cm}$ \\
D2 & $=15 \mathrm{~cm}$ \\
\cline { 2 - 2 } D3 & $=15 \mathrm{~cm}$
\end{tabular}

Figura 4. Espesor de la estructura vial por correlación obtenida

\section{DISCUSIÓN}

Los resultados obtenidos en los ensayos de caracterización de la mina, Tabla 1, cumplen con todas las normas establecidas, lo que indica que la cantera provee agregados adecuados para la construcción de vías en la zona, es decir que se cuenta con un material con condiciones adecuadas. Por otro lado, los valores de C.B.R. obtenidos de los tipos de Bases, Tabla 2, son mayores al $80 \%$ y Sub-bases superiores al $70 \%$, lo que sugiere que es un material muy resistente y es óptimo para la utilización en proyectos viales, ya que el agregado proviene de una mina que se encuentra en el lecho de un río. Los resultados del Módulo Resiliente del material, Tabla 3, se encuentran en el rango propuesto por el tipo de suelo a excepción de Base clase 1 tipo A, Base clase 2 y Base clase 3 que tienen valores un tanto mayores, desde el punto de vista técnico económico es positivo por cuanto en este caso al tenerse un material de mejor calidad se demostró que se pueden bajar los espesores de la estructura del pavimento. Esto se asocia a las características del material y lo hace más resistente.

Los resultados obtenidos de los ensayos de C.B.R. fueron los esperados. El agregado en estudio proviene de una mina de lecho de río y su valor de base y sub-base corresponde a las características del material. En cuanto a los resultados de los ensayos de Módulo Resiliente, los valores se encuentran en el rango de acuerdo al tipo de suelo, resultado asociado a que la 
clasificación SUCS del agregado es de tipo GW, y AASHTO A-1-a.

Los valores de Módulo Resiliente obtenidos, en el caso de sub-base, mediante la correlación obtenida entran en el rango, pero en las bases los números de Módulo Resiliente son mayores a los esperados de acuerdo a la norma AASHOTO 93, lo cual puede ser consecuencia de la resistencia del material, el cual, al ser de lecho de río ubicado en el cantón Santo Domingo de los Tsáchilas, posiblemente haya sufrido una abrasión natural y por consiguiente es un agregado con mejores características.

El diseño vial, utilizando el método AASHTO (93), arrojó espesores típicos de una estructura vial para el TPDA propuesto. La finalidad de esta investigación fue lograr una correlación en la que, a partir de C.B.R., se puedan obtener valores de Módulo Resiliente reales de los agregados de esta mina, y así definir si se cuenta con una optimización de material. En este caso los espesores disminuyeron con respecto a si usáramos las tablas convencionales de correlaciones de las normas. En el caso de la sub-base, los espesores fueron menores a los del diseño original en el cual se utilizaron correlaciones extranjeras, pero por normativas las dimensiones cumplen los mínimos establecidos. La base el espesor se redujo en $5 \mathrm{~cm}$, lo que garantiza que la estructura vial cumplirá con las especificaciones y solicitaciones a las cargas vehiculares provenientes de la carpeta asfáltica. La diferencia de espesores obtenidas entre ambos métodos radica en que el cálculo del diseño por AASHTO (93), toma valores de los ábacos existentes a partir de los C.B.R., no siendo los números necesariamente del agregado a utilizarse. Mientras que, los valores obtenidos por la correlación, son datos apegados a las características propias del agregado, y el segundo diseño optimiza material. El primer diseño es conservador, sin valores reales del agregado. Los dos diseños cumplen con las especificaciones y resisten las cargas de diseño adoptadas y proyectadas a 10 años de acuerdo a las especificaciones de diseño del MTOP. 


\section{CONCLUSIÓN}

La caracterización del material de base y sub-base de la mina en estudio, cumple con todas las normativas ASTM y AASHTO, teniéndose como resultado una mina calificada para la obtención de agregados para diseños viales. Con el logro de una correlación entre el CBR y el Módulo Resiliente real de la mina, se tiene valores reales en el comportamiento de los materiales que se van a usar en la estructura de la vía, teniendo como consecuencia de la optimización de los espesores que, al momento de la construcción, reduce el tiempo de entrega, la cantidad de material y por consiguiente el costo final de la construcción de la vía. Al comparar los diseños obtenidos por el método AASHTO y por la correlación, se tiene un TPDA final bajo y se demuestra que hay una reducción de espesor en la base; pero al tener mayor carga vehicular, es decir un TPDA mayor, se observará una reducción significativa en el espesor de las capas. 


\section{LISTA DE REFERENCIAS}

AASHTO. (1993). AASHTO Guide for Design of Pavement Structures (4th Edition). Estados Unidos.

AASHTO. (2012). AASHTO T307-99 - Determinación del Módulo Resiliente de Suelos y Materiales de Agregado. Washington, Estados Unidos.

ASTM INTERNATIONAL. (2005). ASTM D4318-84-Los métodos de estándar de ensayo para Límite Líquido, Límite Plástico, Índice de Plasticidad de los suelos. West Conshohocken, Estados Unidos.

ASTM INTERNATIONAL. (2006). ASTM C127-Gravedad específica y absorción del agregado grueso. West Conshohocken, Estados Unidos.

ASTM INTERNATIONAL. (2006). ASTM C128-Gravedad específica y absorción del agregado fino. West Conshohocken, Estados Unidos.

ASTM INTERNATIONAL. (2006). ASTM C131-Determinación de la resistencia al desgaste, del agregado grueso de tamaño hasta de $37.5 \mathrm{~mm}$ (1 1/2 pulg), por abrasión e impacto en la Máquina de los Ángeles. West Conshohocken, Estados Unidos.

ASTM INTERNATIONAL. (2006). ASTM C142-97 Determinación de terrones de arcilla y de partículas friables (desmenuzables) en los agregados. West Conshohocken, Estados Unidos.

ASTM INTERNATIONAL. (2006). ASTM C33-Granulometría de agregados. West Conshohocken, Estados Unidos.

ASTM INTERNATIONAL. (2006). ASTM C40-99-Contenido orgánico de la arena. West Conshohocken, Estados Unidos.

ASTM INTERNATIONAL. (2006). ASTM C88-Durabilidad de los agregados a la acción del sulfato de sodio o sulfato de magnesio. West Conshohocken, Estados Unidos. 
ASTM INTERNATIONAL. (2006). ASTM D2419-Equivalente de arena en suelos y agregado fino. West Conshohocken, Estados Unidos.

Camacho, J. (2013). Determinación de la capacidad de soporte C.B.R. del suelo. Valparaíso, Chile: Pontificia Universidad Católica de Valparaíso. Recuperado el 14 de septiembre de 2018.

Gárnica Anguas, P., Pérez García, N., \& Gómez López, J. A. (2001). Módulos de resilencia en suelos finos y materiales granulares. Sanfandila, México.

Herrera Montealegre, M. (2014). Determinación del módulo resiliente de diseño de pavimentos mediante criterrios AASHTO 1993 y 2002. Lima, Perú: Universidad de Piura. Recuperado el 14 de septiembre de 2018

Higuera Sandoval, C. H. (1989). Correlaciones CBR - Módulo Resiliente. Colombia.

Transport Road Laboratory. (1984). Transport Road Laboratory. Lister - New York, Estados Unidos.

Universidad de Chile. (2006). Apuntes de Laboratorio CI44A. Santiago de Chile, Chile.

Villafuerte Bermúdez, L. E., \& Ortega Calle, K. G. (2015). Evaluación estructural de pavimento flexible para suelos de tipo limo arenoso. Quito, Ecuador.

Villalobos Orchard, J. (2016). Departamento de Geología Universidad de Chile. Recuperado el 5 de mayo de 2018, de Las rocas y su proceso de formación: http://www.geologia.uchile.cl/las-rocas-y-sus-procesos-de-formacion

Yánez C, G. (2018). Guía Laboratorio de Pavimentos 2018. Quito, Ecuador. 